\title{
LNT microwave-multiphase transport model for the microwave drying of lignite thin layer
}

Fu, B. A. ${ }^{\mathrm{a}, \mathrm{b} *}$; Chen, M. Q. ${ }^{\mathrm{a}, \mathrm{b}} ; \mathrm{Li}, \mathbf{Q} . \mathrm{H}^{\mathbf{c}^{*}}$

anstitute of Thermal Engineering, School of Mechanical, Electronic and Control Engineering, Beijing Jiaotong University, Beijing 100044, China

${ }^{b}$ Beijing Key Laboratory of Flow and Heat Transfer of Phase Changing in Micro and Small Scale, Beijing 100044, China

${ }^{\mathrm{c}}$ Key Laboratory for Thermal Science and Power Engineering of Ministry of Education, Department of Thermal Engineering, Tsinghua University, Beijng, 100084, P. R. China

*E-mail of the corresponding author: 13116340@bjtu.edu.cn

\begin{abstract}
The LNT microwave-multiphase transport model has been applied to the microwave drying of lignite thin layer. Microwave energy, temperature and moisture distribution were obtained to gain a comprehensive understanding on the heat and mass transfer mechanism of the drying process. The required drying time of experiments decreased by 50,63, 67, and 83\%, respectively, with the power level rising from 119 to $700 \mathrm{~W}$, while that decreased by 60, 72, 76 and 86\%, respectively, for simulation results. The temperature values of the corner and edge of the lignite thin layer were higher than that of the center region, which corresponded to the microwave energy distribution. The moisture ratio profiles, temperature profiles and temperature distribution indicated good agreement between the experimental and simulation results, providing confidence in the modeling approach, which made it possible to obtain the moisture distribution successfully via simulation method
\end{abstract}

Keywords: microwave; lignite; thin layer. 


\section{Introduction}

Lignite is considered as the main energy source for electricity generation and industry feedstocks because of its abundant reservation and cheap price. The high moisture content in lignite leads to low calorific value, low energy utilization efficiency and high transportation cost. Drying prior to pyrolysis, gasification and combustion can improve the utilization efficiencies of the downstream devices. Microwave drying is an efficient method for lignite pre-treatment due to its high drying rate, volumetric heating and precise process control. Apart from experimental studies, numerical modelling is another way to further understand the physics of microwave heating of food. In order to take every possible transport phenomenon for each phase into consideration, and describe the coupled transport mechanisms in a numerical manner, some efforts have been devoted to the modeling of the microwave drying process. With the advancement of computational power and efficient numerical methods, some researchers employed commercial software to provide an exact solution for the Maxwell's equations. Based on the conservation equations elaborated by Whitaker, some researchers derived mechanistic models to highlight the heat and mass transfer characteristics during microwave drying [1]. A coupled electromagnetic, heat and mass transfer model without accounting for pressure driven flow was established by Law et al. [3] to examine the temperature and moisture content distribution in oil palm kernels during microwave drying. They noted that the calculated results fitted the experimental ones, and the cracks of oil palm kernels was prone to occur at the core of the kernel. According to the pumping phenomenon noted by some researchers experimentally, Zhu et al. [4] modified the above model by taking the vapor pressure into account, which would cause moisture diffusion both in liquid and vapor phase. In the case of the microwave drying of potato spheres, good agreement was found between the experimental data and predicted values. In spite of the complete and predictable model developed or modified by the above researchers, the available models still need to be amended with newly discovered physical phenomenon and theory. Swiss scientist Charles Soret discovered that the concentration difference can be built under temperature gradient and the thermo-diffusion phenomenon was referred to as Soret effect [5]. Reversely, the temperature gradient also could be generated by concentration difference, and the diffusion-thermal phenomenon was known as Dufour effect [6]. As a matter of fact, samples can be heated with microwave radiation due to the dipoles orientation [7], and the temperature gradient would be built for the non-uniform microwave energy distribution and energy decay. Corresponding to the dipoles orientation caused by the oscillating electric and magnetic field, Stewart et al. and Lehmann found that liquid crystals showed a uniform rotation under temperature gradient. The thermal polarization phenomenon was also confirmed by Muscatello et al. [8] and Bresme et al. [9] in subsequent numerical calculations, and they found that the water molecules rotation in the direction of temperature gradient would lead to electrostatic field, whose magnitude would scale linearly with the increasing temperature gradient. Based on the complex coupling effects involving the heat and mass transfer and the molecular polarization, the non-equilibrium thermodynamic theory was applied to modify the available models, which can provide more accurate prediction for the coupled heat and mass transfer in porous media during microwave drying.

In the present study, the LNT microwave- multiphase transport model was employed to thin-layer lignite, which is the typical loose structure, to validate the experimental results in terms of moisture ratio and temperature profiles and distribution. The proposed model will 
also be compared with the mechanistic ones with respect to deviation and calculation time. In addition, light is shed on the diffusion-thermal and electric-thermal effect and the microwave-induced coupling transmission mechanism for loose structures during drying process.

\section{Materials and Methods}

\subsection{Problem formulation}

The heat and mass transfer in the lignite thin-layer under microwave irradiation is simplified in Fig. 1. The lignite thin-layer is exposed to the environment where intense microwave energy is introduced from the right side of the oven. The driving force for the liquid water and water vapor diffusion in the thin-layer is the concentration and temperature gradient. The thermal diffusion is caused by the coupling effects of the temperature gradient, the molecular polarization and the liquid water and water vapor diffusion. The molecular polarization is produced by the alternating electromagnetic field and temperature gradient. The heat and mass transfer between the lignite thin-layer and the surroundings only occurs at the top surface.

\subsection{Major hypotheses}

In order to clarify the heat and mass transfer characteristics within the loose structures, the following hypotheses are taken into consideration for the model simplification: (1) local thermodynamic equilibrium exists; (2) the thermodynamic flows scale linearly with the thermodynamic forces; (3) the coupling ratio between the molecular polarization and heat flow presents linear correlation with the average temperature of the sample; (4) all phases are continuous; (5) initial moisture content and temperature within the material are assumed to be uniform; (6) non shrinkage or deformation during drying process; (7) water vapor pressure is shared by all fluid phases; (8) Both the lateral and bottom sides of the lignite thin-layer are insulated; (9) the moisture migration into the surroundings only occurs at the top side of the lignite thin-layer in vapor phase; (10) gravity effect are neglected.

\subsection{Governing equations}

The heat and mass transfer characteristics within the lignite thin-layer during microwave drying can be modelled by the Maxwell equation and the modified mass and energy conservation equations based on the non-equilibrium thermodynamics.

The Maxwell equation is given by [4]:

$$
\begin{aligned}
& \nabla \times E=-j \omega \mu_{0} H \\
& \nabla \times H=j \varepsilon_{0} \varepsilon E \\
& \nabla \times \varepsilon E=0 \\
& \nabla \times H=0
\end{aligned}
$$


where, $E$ is the electric field intensity, $\mathrm{V} \mathrm{m}{ }^{-1} ; H$ the magnetic field intensity, $\mathrm{T} ; \varepsilon$ the complex relative permittivity of lignite thin-layer, which is determined by dielectric constant $\varepsilon^{\prime}$ and dielectric loss factor $\varepsilon^{\prime \prime}, \varepsilon_{0}$ permittivity of free space, $8.85 \times 10^{-12} \mathrm{~F} \mathrm{~m}^{-1}$.

Based on the non-equilibrium thermodynamics, the established mass and energy conservation equations from the former publication [4] can be derived as below and the details can be found in the previous research [10].

The mass conservation equation can be modified as:

$$
\rho_{s} \frac{\partial M}{\partial t}=\left\{\left(\lambda_{g q}+\lambda_{l q}\right) \nabla T\right\}+\nabla\left(K_{g} \nabla p_{g}\right)+\nabla\left(K_{l} \nabla p_{l}\right)
$$

where $\rho_{\mathrm{s}}$ is the density of the lignite thin-layer, $\mathrm{kg} \mathrm{m}^{-3} ; M$ the moisture content (water vapor and liquid water) on dry basis; $\lambda_{g q}$ and $\lambda_{l q}$ the Soret coefficient for vapor and liquid phase, respectively; $K_{g}$ and $K_{l}$ the permeability for vapor and liquid phase, respectively; $p_{g}$ and $p_{l}$ the water vapor and liquid water pressure, respectively, $\mathrm{Pa}$.

The energy conservation equation can be derived as:

$$
\frac{\partial \rho_{\text {eff }} C_{\text {eff }} T}{\partial t}=\nabla(\lambda \nabla T)+\nabla\left\{\frac{a}{\tau}\left(\varepsilon_{0} \chi E_{e q}-P\right)\right\}+h_{g} \nabla\left(K_{g} \nabla p_{g}\right)+h_{l} \nabla\left(K_{l} \nabla p_{l}\right)-\gamma I+Q_{\text {mic }}
$$

where $\rho_{\text {eff }}$ is the effective value of sample denstiy, $\mathrm{kg} \mathrm{m}^{-3} ; C_{p, \text { eff }}$ the effective value of sample specific heat capicity, $\mathrm{J} \mathrm{kg}^{-1} \mathrm{~K}^{-1}[11]$; a the coupling ratio, $\mathrm{V}$; $\tau$ the polarization relaxation time, $s ; h_{g}$ and $h_{l}$ the molar enthalpy of water vapor and liquid water, respectively, $\mathrm{kJ} \mathrm{mol}^{-1} ; \gamma$ the latent heat of evporation, $\mathrm{kJ} \mathrm{kg}^{-1}$; I the evaporation rate, $\mathrm{kg} \mathrm{m}^{-3}$ $\mathrm{s}^{-1} ; Q_{\text {mic }}$ the rate of microwave energy absroption, $\mathrm{W} \mathrm{\textrm {m } ^ { - 3 }}$. $\lambda$ the thermal conductivity involving the pure heat conduction and substance diffusion, $\mathrm{W} \mathrm{m}^{-1} \mathrm{~K}^{-1}$. The details are reported by the previous research.

\subsection{Geometry model}

According to the physical dimensions and shapes of the microwave oven, the geometry model is established with a $460 \times 360 \times 290 \mathrm{~mm}$ cube and three square frustum metal bumps, which can be applied to improve the uniformity of the microwave energy distribution and obtain accurate simulation results. The dimensional details of the metal bumps can be seen in Fig.2. The microwave energy is generated by a coaxial antenna $(10.5 \mathrm{~mm}$ in radius and $12 \mathrm{~mm}$ in height) and introduced into the oven via a rectangular waveguide. 


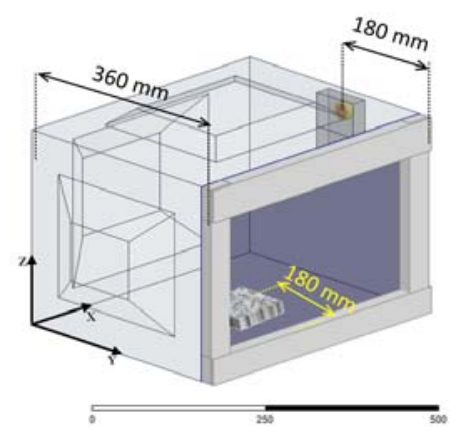

(a) Geometric model.

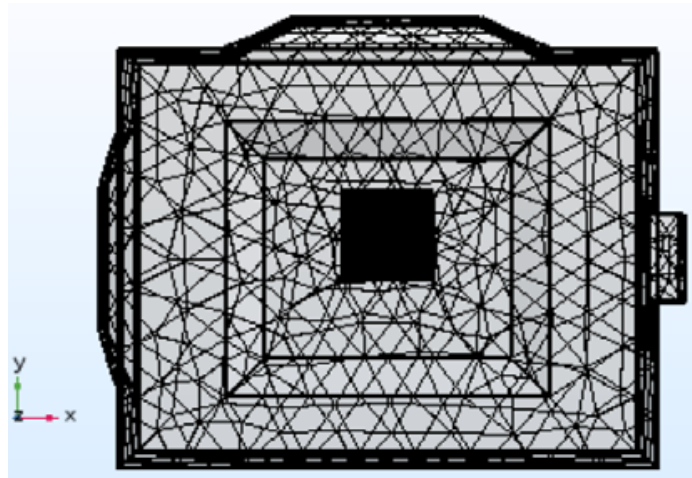

(b) Meshed geometric model.

Fig.1 Geometric model for the microwave dryer setup.

\subsection{Physical model and Initial and boundary conditions}

The physical model, initial and boundary conditions and parameters in the simulation are listed in ref [10], the mathematic and physical models related to coupling mechanism between the heat and mass transfer, and the molecular polarization in a lignite thin layer were developed as above. The governing equations and boundary conditions would be solved by the finite element commercial software COMSOL Multiphysics.

\section{Results and discussion}

\subsection{Drying rates profiles}

The drying and drying rates profiles of experiments and simulations were shown in Fig. 2. From Fig. 2, the microwave power levels had a significant effect on drying time. Time required for microwave drying was shortened with the increase in power level. As shown in Fig. 2 (a), the simulation data fitted the experimental data well with average deviation of $5 \%$ for 119 and $700 \mathrm{~W}$, while $10 \%$ for $231-539 \mathrm{~W}$.

\subsection{Temperature profiles}

The temperature profiles of three points (L, C and R) were shown in Fig. 3. Three periods, detected in the temperature profiles for lignite sample with power level of 119 to $700 \mathrm{~W}$, were the rapid heating period (corresponding to the warm-up and constant rate periods in Fig. 2 (a)), the cooling period (corresponding to the falling rate periods in Fig. 2 (a)) and the final isothermal period, respectively, while only rapid heating period (corresponding to the warm-up, constant rate period and the falling rate period in Fig. 2 (a)) and final isothermal period were detected in the simulation results. 


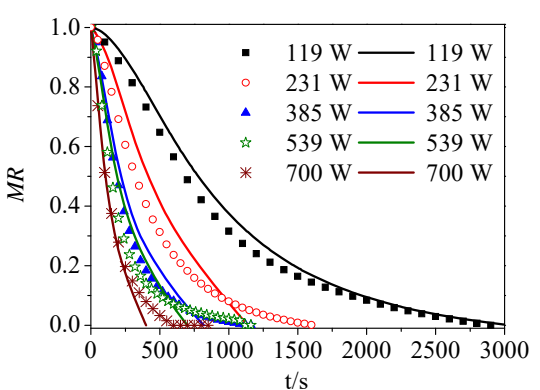

(a) Drying profiles of lignite thin layer

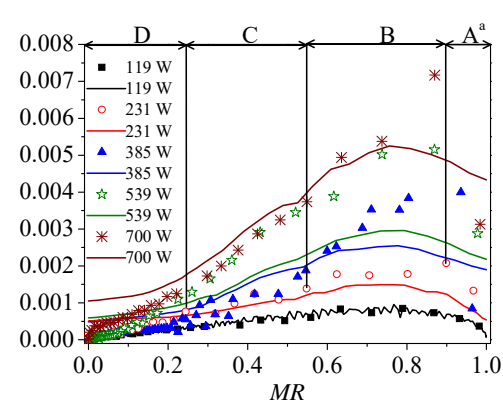

(b) Drying rates of lignite thin layer.

Fig.2. Drying and drying rates of lignite thin layer between 119-700 W. Dots and solid line indicates experimental and simulation data, respectively. $A^{a}-D$ represents the warm-up period, the constant rate period, the first falling rate period and the second falling rate period, respectively.

The comparison of temperature between the experimental and simulation data for five power levels was illustrated in Fig. 3. The simulation data fitted the experimental data well with average deviation of $5 \%$ for $119 \mathrm{~W}$ and about $10 \%$ for $231-539 \mathrm{~W}, 15 \%$ for $700 \mathrm{~W}$.

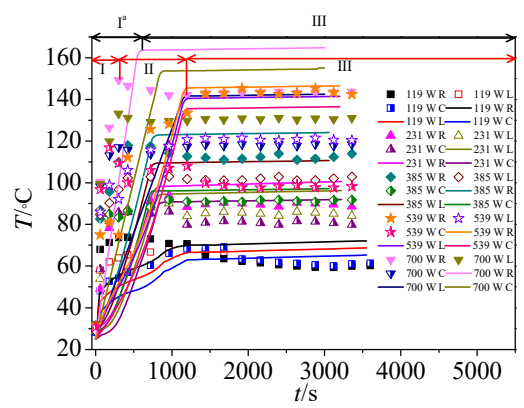

Fig.3. Temperature profiles of lignite thin layer between 119-700 W. Dots and solid line indicates experimental and simulation data, respectively. $I^{a}$ : the heating up period; II: the cooling down period; III: the isothermal period.

\subsection{Microwave energy and temperature distribution}

The temperature distribution of experiments and simulations at $385 \mathrm{~W}$ were shown in Fig. 4. As illustrated in Fig. 4, the non-uniform temperature distribution of sample surface was evidenced by experiments and simulation results with the moisture ratio of 0.8 and 0.2 at $385 \mathrm{~W}$. The temperature distribution obtained by simulation method possessed similar trend with experimental results, which verified the calculation results of temperature distribution based on numerical simulation. 
Fu, B. A.; Chen, M. Q.; Li, Q. H.
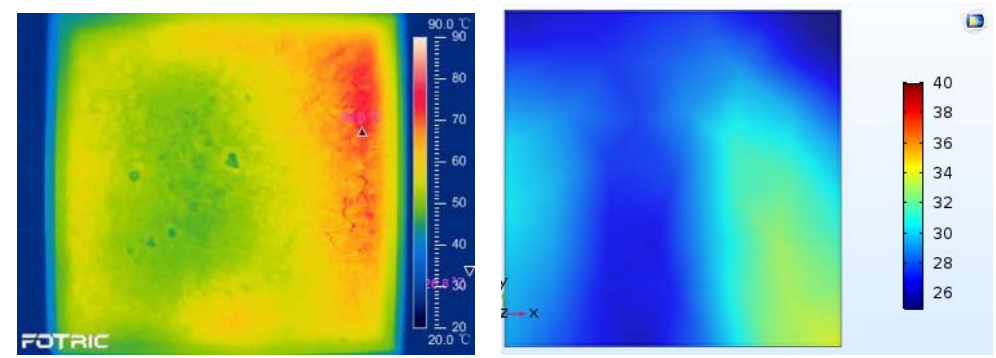

(a) Experimental results. (b) Simulation results.

$\mathrm{MR}=0.8$

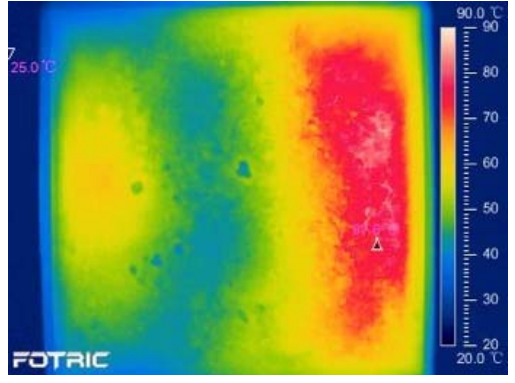

(a) Experimental results.

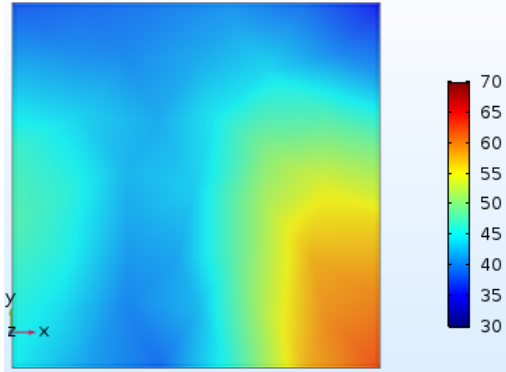

(b) Simulation results.

$\mathrm{MR}=0.2$

Fig.4. Temperature distribution of experiments and simulation.

\subsection{Moisture distribution}

Moisture distribution in lignite thin layer at $385 \mathrm{~W}$ was illustrated in Fig. 5. As shown in Fig. 5, the moisture content was lower at the right and left regions in the lignite thin layer, which corresponded to the temperature distribution.

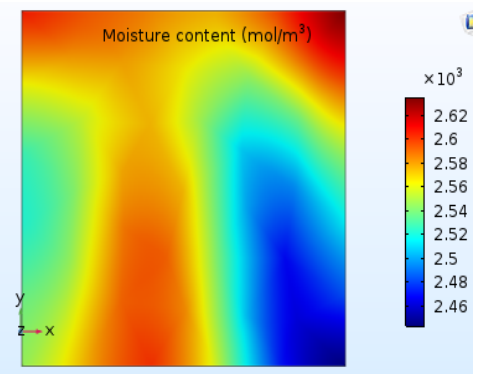

Fig.5. Moisture content distribution in sample at $385 W(M R=0.8)$. 


\section{Conclusions}

The LNT microwave-multiphase transport model has been applied to simulate the microwave drying behavior of lignite thin layer successfully. Microwave energy, temperature and moisture distribution were obtained to gain a comprehensive understanding on the heat and mass transfer mechanism of the drying process. The simulation data of the moisture ratio fitted the experimental data well with average deviation of $5 \%$ for 119 and $700 \mathrm{~W}$, while $10 \%$ for $231-539 \mathrm{~W}$. The average deviation of temperature between the simulation and experimental data were $5 \%$ for $119 \mathrm{~W}$ and about $10 \%$ for $231-539 \mathrm{~W}$, while $15 \%$ for $700 \mathrm{~W}$. The coupled electromagnetics and multiphase porous media made it possible to obtain the moisture distribution via simulation method. Further investigation on the moisture distribution will be carried out via MRI experiments to verify the simulation result.

\section{Referrence}

[1] A.K. Datta, Porous media approaches to studying simultaneous heat and mass transfer in food processes. I: Problem formulations, J. Food Eng. 80 (1) (2007) 80-95.

[2] K. Pitchai, S.L. Birla, J. Subbiah, D. Jones, H. Thippareddi, Coupled electromagnetic and heat transfer model for microwave heating in domestic ovens, Journal of Food Engineering 112 (1) (2012) 100-111.

[3] M.C. Law, E.L. Liew, S.L. Chang, Y.S. Chan, C.P. Leo, Modelling microwave heating of discrete samples of oil palm kernels, Applied Thermal Engineering 98 (2016) 702 726.

[4] H. Zhu, T. Gulati, A.K. Datta, K. Huang, Microwave drying of spheres: Coupled electromagnetics-multiphase transport modeling with experimentation. Part I: Model development and experimental methodology, Food and Bioproducts Processing 96 (2015) 314-325.

[5] M.A. Rahman, M.Z. Saghir, Thermodiffusion or Soret effect: Historical review, Int. J. Heat Mass Transfer 73 (2014) 693-705.

[6] D. Pal, H. Mondal, Influence of chemical reaction and thermal radiation on mixed convection heat and mass transfer over a stretching sheet in Darcian porous medium with Soret and Dufour effects, Energy Convers. Manage. 62 (2012) 102-108.

[7] B.A. Fu, M.Q. Chen, Y.W. Huang, H.F. Luo, Combined effects of additives and power levels on microwave drying performance of lignite thin layer, Drying Technology 35 (2) (2017) 227-239.

[8] J. Muscatello, F. Romer, J. Sala, F. Bresme, Water under temperature gradients: polarization effects and microscopic mechanisms of heat transfer, PCCP 13 (44) (2011) 19970-19978.

[9] F. Bresme, A. Lervik, D. Bedeaux, S. Kjelstrup, Water Polarization under Thermal Gradients, Phys. Rev. Lett. 101 (2) (2008) 020602.

[10] B.A. Fu, M.Q. Chen, Q.H. Li, J.J. Song, Non-equilibrium thermodynamics approach for the coupled heat and mass transfer in microwave drying of compressed lignite sphere, Appl. Therm. Eng. 133 (2018) 237-247.

[11] A.K. Datta, Porous media approaches to studying simultaneous heat and mass transfer in food processes. II: Property data and representative results, J. Food Eng. 80 (1) (2007) 96-110. 